strongly of drink'. In contrast to the Maudsley Emergency Clinic, $21 \%$ presented during the day, $63 \%$ during the evening ( 5 p.m. -12 midnight), and only $16 \%$ overnight (12 midnight- 10 a.m.).

The point I want to highlight is that of the 57 patients who were lodged or 'guested' overnight in the centre, $73 \%$ attended the next available clinic at the Alcohol Treatment Unit in contrast to only $46 \%$ of the 61 patients not lodged. There was no evidence that the junior doctors chose for lodging only those patients likely to attend. The most likely explanation is that lodging favourably influenced attendance. Why this was so may have many reasons ranging from proximity and practical ease of access, to the response of disturbed dependent persons to a 'holding environment'.

Recently the pessimism and gloom about the prognosis for those who abuse alcohol or become dependent on it at stages during their lives is lessening. It would be a pity if a response or lack of response to such persons inhibited a process that may lead to beneficial life changes. Whether this is so at the Maudsley Emergency Clinic, a setting which many would hope to emulate, is a question worth considering.

Cassel Hospital
Richmond, Surrey

KeVIN Healy

REFERENCE

'McGennis, A. J., O'Callaghan, J., Tedders, J. G. et al (1980) Screening psychiatric admissions: 6-months' experience in the assessment centre of a large urban psychiatric hospital. Journal of the Irish Medical Association, 73, 351-356.

\section{Dr Haw and colleagues reply}

DeAR SIRS

We feel the Maudsley Emergency Clinic's policy of turning drunk patients away and asking them to return for assessment when sober is both humane and sensible. If every client who presented drunk and claimed drinking problems was admitted the clinic's resources would be overwhelmed. Asking people to return when sober is a small test of motivation and selects those clients amongst this difficult group who show some inclination to stop drinking.

The project described by Dr Healy in his letter is an interesting pilot study but the assertion, "There was no evidence that the junior doctors chose for lodging only those patients likely to attend", needs to be validated by a randomised study. Thus at present we see no justification for a change to existing policy.

St Bernard's Hospital

C. M. HaW Southall, Middlesex

St Augustine's Hospital

C. P. LANCEley

Canterbury, Kent

S. J. VICKERS

Imperial College

London SW7

\section{The College and NHS cuts}

DeAr Sirs

Presently, we are seeing a general turmoil in the National Health Service due to the Government's policy of cutting services and closing long-stay hospitals. From the psychiatric point of view, the main impact has been on psychogeriatric and mental handicap hospitals. The most worrying aspect is the closure of hospital wards before the opening of comparative treatment facilities in the community which has resulted in suffering for patients and their families.

Recently, three Royal College Presidents gave their views on the Government's National Health Service policies. Perhaps it would be helpful if a fourth member, the President of the Royal College of Psychiatrists, joined the team of protesters. It would also be more relevant as psychiatry as a whole is taking the brunt of the changes which alter its long-standing functions and practices.

U. J. DEY

Brockhall Hospital

Old Langho, Nr. Blackburn

Lancashire

DEAR SIRS

We have been interested to observe the public comments of the Presidents of the Royal Colleges of Surgeons, Physicians and Obstetricians and Gynaecologists on the parlous state of the National Health Service. We have been surprised by the absence of any such activity from the officials of our College. Is this a tactical manoeuvre suggested by our recently appointed public relation consultants?

JONATHAN LOVETT

Countess of Chester Hospital

Chester

Department of Psychiatry

LISETTA LOVETT

University of Liverpool

The President writes:

Public activity is, to some extent, a matter of timing. The letter from the three Presidents was closely followed by the delivery of a petition to Downing Street, by a delegation in which I took part. This activity may have contributed to the release of a small amount of extra money.

The longer term requires less public, but equally forcible, activity. We have pointed out, with good evidence, to the DHSS that funding has been diverted from mental health services to the acute sector. This may be more publicly discussed in due course, but the point has been made. All this relates first, to the possible extra funding for the NHS in 1988-1989 from the budget and secondly, to the longer term plans for the NHS. The government has been repeatedly advised that people with persistent disabilities and recurrent illness fare very badly from private insurance schemes. Our present concern is with the (unpublished) Griffiths report. Some of its recommendations sound very worrying. 